\title{
Crystal Nucleation by Laser-Induced Cavitation
}

\author{
Anamaria Soare, ${ }^{,+}$Rory Dijkink, ${ }^{\ddagger}$ Marcos Rodriguez Pascual, ${ }^{\dagger}$ Chao Sun, ${ }^{\ddagger}$ Peter W. Cains, ${ }^{+}$ \\ Detlef Lohse, ${ }^{\neq}$Andrzej I. Stankiewicz, ${ }^{\dagger}$ and Herman J. M. Kramer ${ }^{+}$
}

${ }^{\dagger}$ Intensified Reaction and Separation Systems, Delft University of Technology, 2628 CA Delft, The Netherlands

${ }^{\ddagger}$ Physics of Fluids, University of Twente, 7522 NB Enschede, The Netherlands

\begin{abstract}
High-speed and high-resolution photography have been used to investigate the relationship between creation, expansion, and collapse of a vapor cavity induced by a 6 ns laser pulse and the subsequent nucleation of crystals. A thin layer of supersaturated aqueous solutions of $\left(\mathrm{NH}_{4}\right)_{2} \mathrm{SO}_{4}$ and $\mathrm{KMnO}_{4}$ was confined between two glass plates with a separation of 50 and $100 \mu \mathrm{m}$. The expansion and collapse of the laser-induced vapor bubble occurred over a total time scale of $200 \mu \mathrm{s}$, while the first identifiable crystal appears one second after the laser
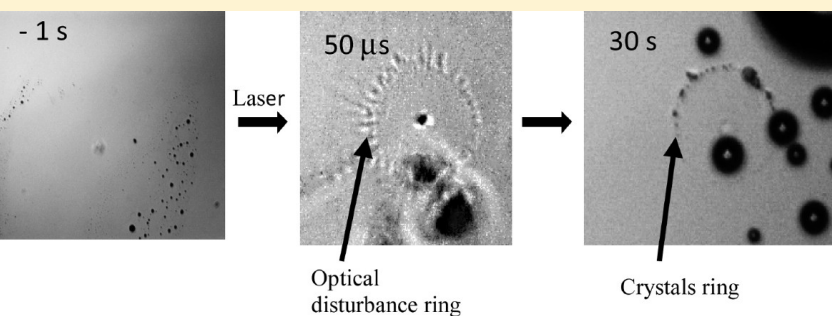

pulse. Crystals were observed to form on a ring with a diameter of $70 \mu \mathrm{m}$ centered in the focal point of the laser. The ring is preceded by an optical disturbance observed through the cavity around $30-50 \mu$ s after the laser pulse and vapor cavity formation. This ringshaped optical disturbance originates from changes in refractive index induced by crystal nuclei formation. The formation of the nuclei most probably coincides with the formation of the bubble, when the rate of evaporation and the supersaturation are at their maxima. Apparently, it takes the nuclei around $30-50 \mu$ s to grow to a particle size with a visible optical disturbance.
\end{abstract}

\section{INTRODUCTION}

The effectiveness and reproducibility of crystallization is often limited by crystal nucleation, ${ }^{1}$ a stochastic process that is generally not well understood and that can often show considerable variability even under constant and well-controlled conditions. ${ }^{2}$ A number of expedients can be employed to overcome these difficulties, the most common of which are seeding and the application of ultrasound energy. ${ }^{3}$ Ultrasound in the so-called "power" frequency range of $20-100 \mathrm{kHz}$ has been shown to induce nucleation in a wide range of crystallization processes, ${ }^{4}$ but its effectiveness is by no means universal. Most of the observed physical chemical effects of power ultrasound have been attributed to sonically induced cavitation (bubble or void formation $)^{5}$ and in particular to the collapse of cavitational voids during the compression phase of the sonic cycle. ${ }^{6}$ It is generally assumed that crystal nucleation is associated with such cavitational collapses, but the mechanistic pathway by which cavitation and collapse lead to crystal nucleation has not been satisfactorily explained. An alternative explanation of ultrasonically induced nucleation ${ }^{7}$ assumes that the bubble surface created in cavitation acts as a foreign particle, so that nucleation is heterogeneous. However, results of experiments in which gas bubbles are introduced in place of ultrasound show that this nucleation is not sensitive to the application time of the gas.

Experimentally, it is very difficult to locate and monitor a single cavitation event in an ultrasonic field. ${ }^{8}$ Instead, we have examined in detail the relationship between the growth/collapse of a cavity and crystal nucleation using a focused laser pulse to induce a single cavity in a supersaturated solution at a specific, predetermined location. However, we note that for laser-induced cavitation the bubble dynamics 9 and the subsequent physical effects differ from those for ultrasonic cavitation. Whereas in the latter case the bubble mainly consists of gas, laser induced bubbles mainly consist of vapor, and growth and shrinkage are controlled by evaporation, condensation, and thermal diffusion which is much faster than mass diffusion, so that is inappropriate to speak of some equilibrium bubble size. We will discuss these differences between gas and vapor bubbles in more detail below.

Laser irradiation has been reported to induce crystal nucleation on its own by a number of mechanisms, some of which involve cavitation ${ }^{10}$ and some of which do not. ${ }^{11}$ The crystallization of anthracene from solution in cyclohexane was triggered by a single femtosecond laser pulse of energy above the threshold for cavity formation $(3.1 \mu \mathrm{J})$ and occurred at the bubble surface. ${ }^{10}$ A similar femtosecond pulse was used to induce the nucleation of hen egg-white lysozyme (HEWL), ${ }^{12}$ but here nucleation was only observed $24-48 \mathrm{~h}$ after irradiation and it is not known whether cavitation occurred. In so-called "nonphotochemical" laser-induced nucleation or NPLIN, an expanded laser beam is employed, ${ }^{11}$ and presumably cavitation is avoided. Here, the induction of nucleation is ascribed to the optical Kerr effect, in which the polarized electromagnetic vectors of the beam align polar functions within the crystallizing molecule and thus induce ordering in the nucleating clusters. To support this hypothesis, a switch in the polymorphs of glycine that crystallize from solution was observed depending on

\footnotetext{
Received: January 1, 2011

Revised: $\quad$ March 24, 2011

Published: April 05, 2011
} 
whether the beam is linearly or circularly polarized. ${ }^{13}$ Single laser pulses of $6-7$ ns duration were reported to lead to crystal nucleation in supersaturated solutions of $\mathrm{KCl}$ according to this mechanism, ${ }^{14}$ and this method was also used to induce spatially controlled crystallization of $\mathrm{KCl}$ in an agarose gel. ${ }^{15}$ It has also been shown that ice nucleation in supercooled water can be initiated by the optical breakdown induced by a focused laser pulse of $1064 \mathrm{~nm}$ wavelength. ${ }^{16}$ Even if the detailed mechanism is not proven yet it is argued that homogeneous nucleation in the compressed liquid phase is a plausible explanation of the effect.

Here we report the direct observation of nuclei formation around a single forming, expanding and collapsing cavity, created by a focused laser pulse. By using a thin layer of supersaturated solution between two glass slides, it was possible to obtain wellfocused two-dimensional (2-D) digital images of the bubble growth, collapse, and the resulting nucleation.

\section{EXPERIMENTAL SECTION}

Experiments were carried out using aqueous solutions of two simple inorganic salts: $\left(\mathrm{NH}_{4}\right)_{2} \mathrm{SO}_{4}$ and $\mathrm{KMnO}_{4}$. Attempts to extend the range of case studies to organic solutes and solvents (methanol, ethanol) were hampered by the solvent volatility and possible thermal degradation. With $\left(\mathrm{NH}_{4}\right)_{2} \mathrm{SO}_{4}$ solutions, the operating temperature was $22.5^{\circ} \mathrm{C}$ and relative supersaturation levels of 0.2 and $0.4 \%$ were employed; with $\mathrm{KMnO}_{4}$ the operating temperature was $29^{\circ} \mathrm{C}$ and the relative supersaturations were 7, 14, and $21 \%$ (see Results). Magenta ink was added to

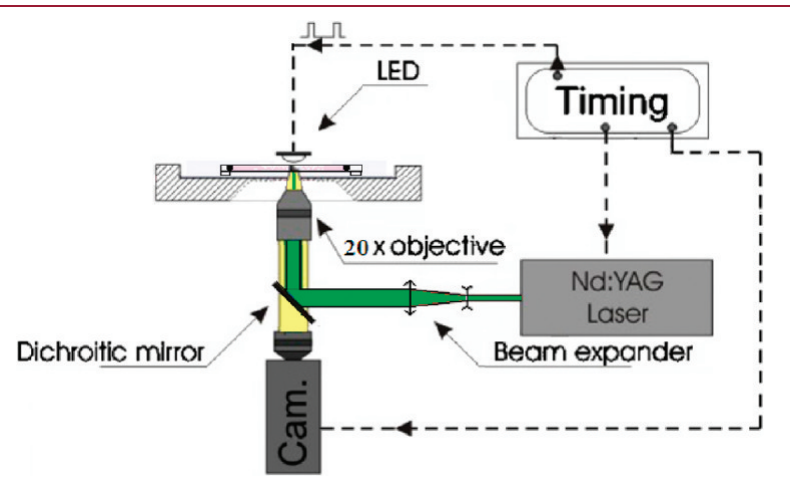

Figure 1. Experimental setup for observing laser-induced cavitation and nucleation. the $\left(\mathrm{NH}_{4}\right)_{2} \mathrm{SO}_{4}$ solutions to facilitate the absorption of the laser energy in the solution and hence the creation of the cavitation bubble.

The liquid was placed in between two glass plates with a 50 or $100 \mu \mathrm{m}$ gap; see Figure 1. A Nd:YAG 6 ns laser pulse (wavelength $532 \mathrm{~nm}$ ) of energy $0.05-0.5 \mathrm{~mJ}$ (Solo PIV, New Wave, Fremont, CA, USA) was then focused by a $20 \times$ objective lens to the center of the liquid to create a cavitation bubble. The bubble grows explosively, rapidly exceeding the distance between the glass plates, thus appearing two-dimensional. A single frame high-sensitive camera (PCO) was also employed, in combination with a $1 \mu$ s light pulse from a high intensity LED (Seoul P7), to image the bubble and the crystals that subsequently formed. A digital delay generator (model 555, Berkeley Nucleonics Corp., CA, USA) was used to synchronize these cameras and the laser. The motion of the bubble was recorded by a high-speed camera (HPV-1, Shimadzu Corp., Japan). The time lapse between pictures was $4 \mu$ s. Illumination for the camera was provided by a fiber lamp (Olympus ILP-1) emitting white light, which was redirected by a fiber optic arm, passing through the dichroitic mirror filter to the camera.

\section{RESULTS}

Figure 2 shows the formation, growth, and collapse of the cavitation bubble for a typical experiment with $\left(\mathrm{NH}_{4}\right)_{2} \mathrm{SO}_{4}$ solution. In (a) at $4 \mu \mathrm{s}$ after the laser pulse, the vapor bubble is already formed at the focal point of the pulse. The bubble is initially a small sphere that expands spherically until it occupies almost the entire gap between the two slides (a), after which preferential growth in the radial direction begins and the shape of the bubble changes to that of a cylindrical slice (b), (c), and (d). Two very thin liquid films remained on the walls, indicating wetting of the hydrophilic surface. Then the bubble collapse (e), (f) and (g), leaving small gas bubbles behind (black circles in (g) and $(\mathrm{h}))$. These are noncondensable air bubbles. In the order of minutes later crystals were observed as seen in (h).

Figure 3 a shows the evolution of the bubble radius during formation and collapse, taken from analysis of the photographic images. The bubble expands quickly and shrinks more slowly after reaching its maximal size, which means that the (absolute) velocity of the interface, Figure $3 b$, is greater during the expansion.

The absorbed laser pulse superheats the liquid which finally evaporates explosively, leading to a shock wave emission. The volume of the vapor bubble then expands with a velocity smaller than the shock wave. This interface velocity is given in Figure $3 \mathrm{~b}$.

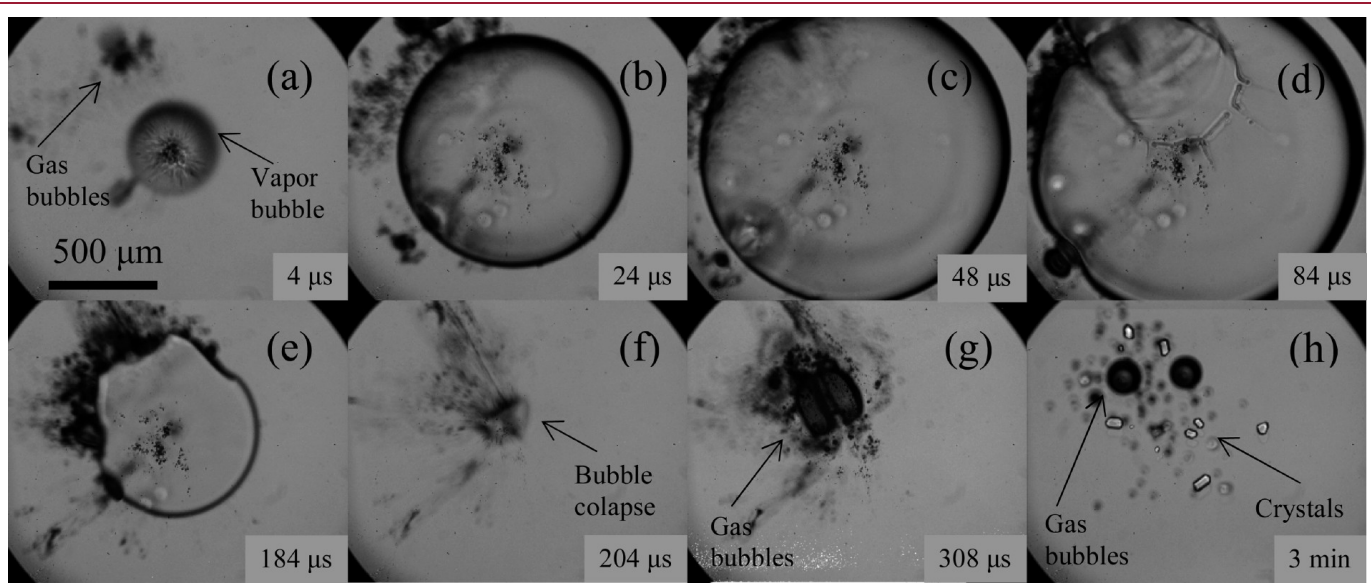

Figure 2. Evolution of cavitational bubble with time, $\left(\mathrm{NH}_{4}\right)_{2} \mathrm{SO}_{4}$ solution, $0.4 \%$ supersaturation, $100 \mu \mathrm{m}$ gap size. After the laser pulse, the bubble forms rapidly by evaporation, grows, and collapses, over a time scale of $200 \mu \mathrm{s}$, leaving behind crystals which in time grow to sizes of tens of micrometers. 

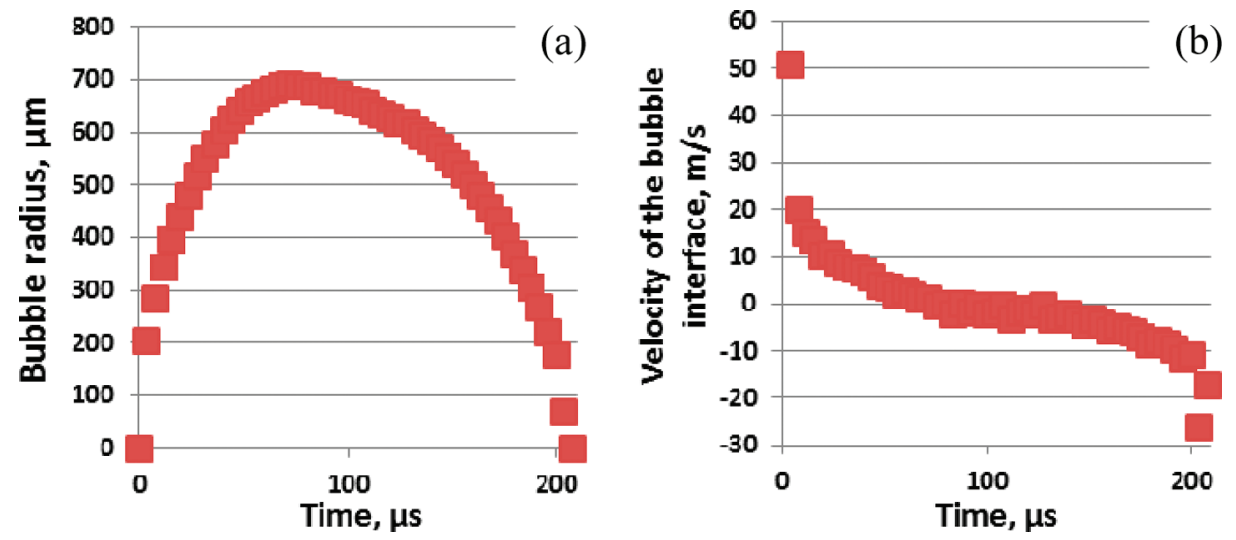

Figure 3. Evolution of (a) the bubble radius and (b) the bubble interface velocity with time. Experimental conditions as in Figure 2 . The focused laser beam width is $20 \mu \mathrm{m}$.

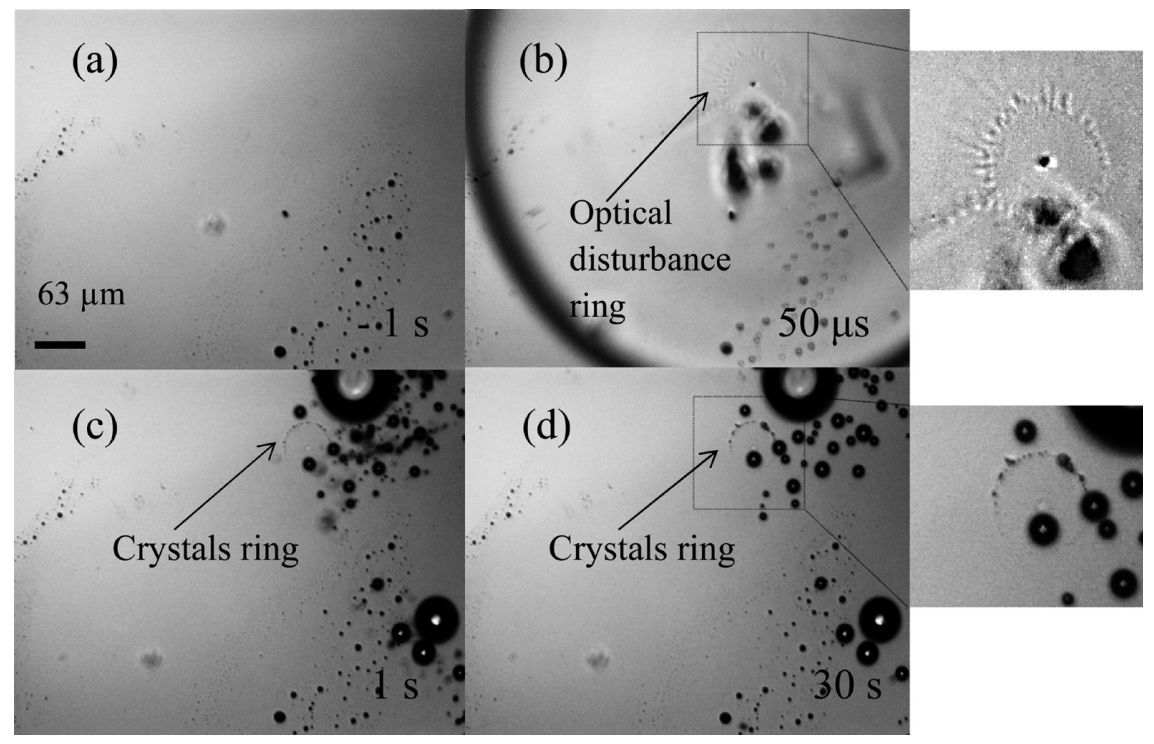

Figure 4. $(\mathrm{a}-\mathrm{d})$ High-resolution images during cavity formation and nucleation of $\left(\mathrm{NH}_{4}\right)_{2} \mathrm{SO}_{4}$ solution, $0.2 \%$ supersaturation, $50 \mu \mathrm{m}$ gap size.

Images taken with the high-resolution camera at key points in the sequence of cavity evolution and crystal nucleation in Figure 2 are shown in Figure 4. Figure 4a shows the clear solution prior to firing the laser; the small round black dots are air bubbles. Figure $4 \mathrm{~b}$ shows the expanding cavitation bubble at the point corresponding to Figure $2 \mathrm{c}, \sim 50 \mu \mathrm{s}$. Close examination reveals a thin ring-shaped optical disturbance, indicated by the arrow, surrounding the focal spot of the laser and visible through the bubble. One second after the collapse of the bubble, Figure $4 c$, a ring of small crystals may be seen in exactly the same place as the disturbance in Figure $4 \mathrm{~b}$. After a further $30 \mathrm{~s}$, the crystals can be seen to have grown, Figure 4d.

Figure 5 shows a zoom on the area close to the focal point of the laser. The disturbance through the bubble can clearly be seen in Figure 5a. The diameter of the ring is about $70 \mu \mathrm{m}$. The subsequent ring of crystals in the same place in Figure $5 \mathrm{~b}$ has the same diameter. In the center of Figure $5 b$, a minute crack in the lower glass slide can be seen.

This was caused by the thermal shock arising from the absorption of laser energy by the glass. Even with the addition of magenta ink, it was difficult to obtain a cavitation bubble without creating such small cracks in the support slide.
Two questions arise: Why are the crystals formed in a ring structure and what physical process determines the final radius of the ring? We did not observe any clear relation between the absorbed laser energy and the radius of the ring of crystals. The crystals are most probably formed at the liquid-vapor interface immediately after the bubble formation, and are then - thanks to inertia - shifted outward with the expanding vapor-liquid interface into the cold supersaturated liquid where they further grow. When moving outward they thus accumulate mass and inertia so that their movement more and more decouples from that of the less dense liquid. Upon collapse, the crystals do not follow the vapor liquid interface any more (similar to gas-liquid interface-driven particles ${ }^{17}$ ) but remain at some radius.

Further experiments with $\mathrm{KMnO}_{4}$ solution were carried out to ensure that we were observing nucleation caused by laser irradiation and not by secondary nucleation due to small shards of glass detached by cracking or to foreign ions possibly formed by photochemically degradation of the ink. The intense coloration of this solution and the consequent high absorption at the laser frequency ensured that no damage to the glass slides occurred, as we established by careful examination. The photo 
graphic images show the same sequence of events as those presented above, but they are generally less clear because of the coloration of the solution. Figure 6 shows a sequence of cavitation and nucleation events with $\mathrm{KMnO}_{4}$ that are similar to those recorded above with $\left(\mathrm{NH}_{4}\right)_{2} \mathrm{SO}_{4}$.

In these experiments in which no ink was added, there were no problems in obtaining cavitation bubbles without any cracking in the glass slides, and the crystals were again observed one second after the laser pulse. However, with $\mathrm{KMnO}_{4}$ solutions, crystals were obtained after cavity formation only in solutions where the initial supersaturation was higher than $7 \%$. For solutions with supersaturation of $7 \%$ or lower, crystals were not obtained after a single laser pulse but could be obtained after several sequential pulses. The number of pulses required in these cases was between 5 and 20, with $1 \mathrm{~s}$ time lapses between shots. If the repetition rate of the laser pulses increased 2-3 times the crystals appeared after fewer pulses; if the repetition rate decreased $2-3$ times, more pulses were required to create crystals. This is believed to have occurred because the evaporation arising from a single pulse was
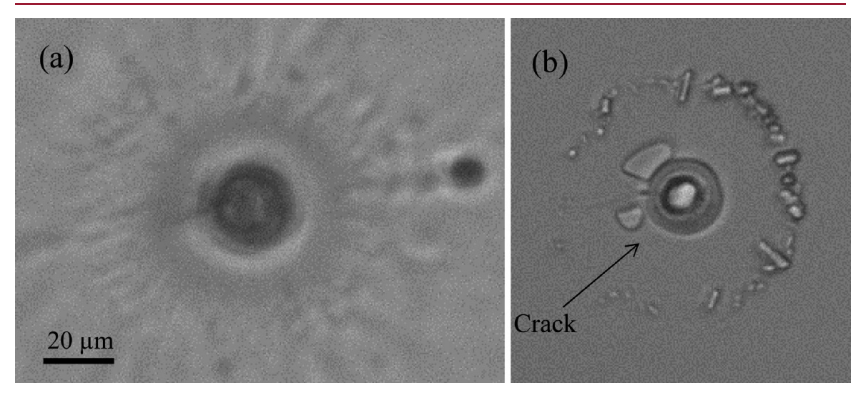

Figure 5. Zoom view of (a) the optical disturbance around the laser focal spot (as Figure 4b), (b) the subsequent ring of crystal nuclei. A minute crack may be seen in the lower glass slide, shown by the arrow. The scale bar in (a) also refers to panel (b) which is shown at the same scale. insufficient to create nucleation, given that a corresponding local temperature rise will decrease the supersaturation. After multiple pulses, eventually the evaporation will be sufficient for crystal nucleation to take place. Each pulse induces cavitation, and after each collapses the local temperature of the liquid increases; thus less energy is used for heating the liquid and more energy is used for evaporation. With $\left(\mathrm{NH}_{4}\right)_{2} \mathrm{SO}_{4}$, because the temperaturesolubility curve is very flat, the effect of the small amount of water evaporated by the pulse will not be compensated to the same extent by a solubility increase that results from a rise in temperature.

With $\left(\mathrm{NH}_{4}\right)_{2} \mathrm{SO}_{4}, 10-30$ crystals were formed per laser pulse and with $\mathrm{KMnO}_{4}$ only $1-5$ crystals were obtained for each event. This may also be due to the balancing effects of evaporation and temperature increase as described above, but it may also reflect the narrower metastable zone of $\left(\mathrm{NH}_{4}\right)_{2} \mathrm{SO}_{4}$.

At very high supersaturation (21\%), very small particles formed at the glass surface immediately after the solution was introduced into the gap and prior to laser pulsing. Without laser pulses these grew very slowly, with no increase in size after 15 min. However, these particles differed in appearance and in color to those of $\mathrm{KMnO}_{4}$, and they were difficult to remove from the glass after the experiment. We believe they were $\mathrm{MnO}_{2}$ deposits arising from decomposition rather than crystals of $\mathrm{KMnO}_{4}$ and that they do not affect the crystallization of $\mathrm{KMnO}_{4}$. A few large $\mathrm{KMnO}_{4}$ crystals were observed around $1 \mathrm{~s}$ after a single pulse, and more appeared after subsequent pulses. In this case, around 3-7 more $\mathrm{KMnO}_{4}$ crystals were obtained per laser pulse. This increase in the number of crystals is a consequence of the higher initial supersaturation of the solution.

\section{DISCUSSION}

We have shown by direct observation that the creation of a laser-induced cavity in a supersaturated solution results in the

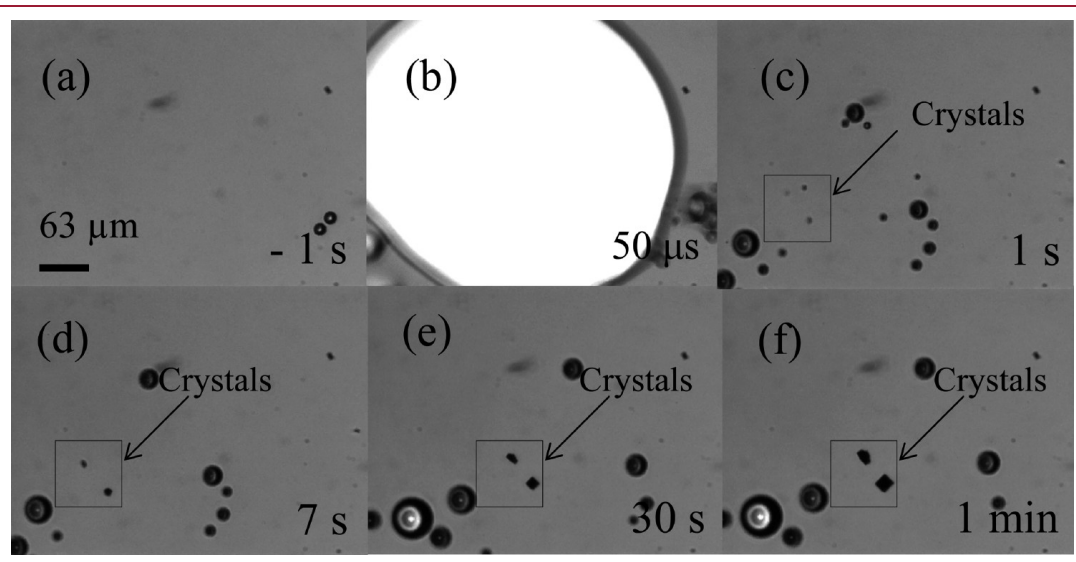

Figure 6. (a-f) $\mathrm{KMnO}_{4}$ nucleation and growth, $14 \%$ initial supersaturation, $50 \mu \mathrm{m}$ gap size, $0.06 \mathrm{~mJ}$ energy input.

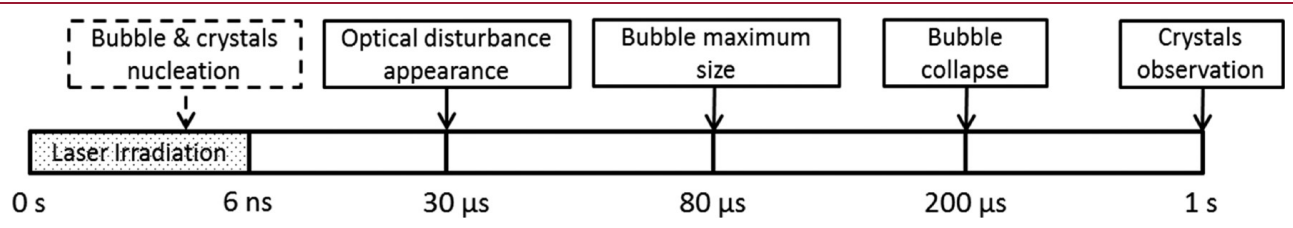

Figure 7. Time line diagram for crystal nucleation by laser-induced cavitation experiments. The bubble and crystals nucleation are in a dashed rectangle because it is not known when during the first 6 ns they actually take place. 
nucleation of crystals in a ring around the laser focus, where the creation and collapse of the cavity occur. In the cases investigated, the growth and collapse of the cavity take place over time scales of $\sim 200 \mu$ s, while the appearance of visible and identifiable crystals requires on the order of $1 \mathrm{~s}$, although observable optical disturbances were already present after $30-50 \mu$ s. The formation of nuclei occurs on a ring centered on the center of the cavity, in the same position as the optical disturbance occurs during the development of the cavity. Figure 7 shows a time line diagram representing bubble and nuclei evolution.

Essentially the same results were observed with $\left(\mathrm{NH}_{4}\right)_{2} \mathrm{SO}_{4}$ and $\mathrm{KMnO}_{4}$ solutions, but with $\left(\mathrm{NH}_{4}\right)_{2} \mathrm{SO}_{4}$ the nucleation effects are much more clearly exhibited. With $\mathrm{KMnO}_{4}$, higher supersaturations were needed for nucleation to occur, a smaller number of nuclei were created, and in some cases multiple laser pulses were required to create nuclei. The metastable zone width for the crystallization of $\mathrm{KMnO}_{4}$ from aqueous solution was not measurable accurately under the conditions of the experiment, because of the solution coloration, but the measurements did show that the zone was much wider than that for $\left(\mathrm{NH}_{4}\right)_{2} \mathrm{SO}_{4}$ solutions. ${ }^{18}$ This can explain why higher supersaturation levels had to be employed; no nucleation could be induced below $7 \%$ supersaturation.

Recent investigations of the formation and collapse of a vapor cavity under laser irradiation in a microtube filled with liquid (water with an added red dye ${ }^{9,19}$ reported similar vapor bubble dynamics to those in Figures 2 and 3 of this work, namely, rapid expansion and somewhat slower contraction and collapse, and evidence of surface wetting as the cavity expanded. In those papers, the expansion and contraction of the cavities were successfully modeled, taking both hydrodynamic and thermal effects into account. The calculations indicated that temperature transients of $180{ }^{\circ} \mathrm{C}$ occurred on impulse at the start of cavity formation, falling rapidly to $70-80^{\circ} \mathrm{C}$ at the point of maximum expansion and to $50-60^{\circ} \mathrm{C}$ at the point of collapse.

In most cases including both $\left(\mathrm{NH}_{4}\right)_{2} \mathrm{SO}_{4}$ and $\mathrm{KMnO}_{4}$, temperature transients are unlikely to result directly in crystal nucleation because solubility increases with temperature and the supersaturation driving force to nucleate will consequently decrease. Similarly, also in ultrasonically induced nucleation thermal effects in the liquid are limited due to the large thermal capacity of water. ${ }^{20}$ For this reason, alternative explanations of ultrasonically induced nucleation have been sought in terms of the pressure transient or shock wave that accompanies collapse, ${ }^{21}$ but these have not yet been examined rigorously.

The position at which crystals are eventually detected is directly related to the optical disturbance observed through the vapor bubble around 30-50 $\mu$ s after the laser pulse, Figure 5. At present, this disturbance has not been characterized completely but firmly suggests changes in refractive index induced by nuclei formation at the point of maximum rate of vaporization at the start of bubble formation during the laser pulse. From the moment of crystal nucleation, it takes around $30-50 \mu$ s for the nuclei to grow large enough to generate an optical disturbance.

Experiments in a three-dimensional (3-D) cuvette were also performed, but those experiments did not bring new insights regarding the mechanism of cavitation induced crystal nucleation, as the crystals were observed only at the end of the experiments at the bottom of the cuvette.

This investigation was undertaken primarily to understand the mechanism of ultrasonic nucleation, by relating cavitational events and collapse to the phenomenon of crystal nucleation. While there are parallels in the characteristics and behavior of laser- and ultrasonically induced cavitation, the sequence of events differs. With laser induction, a shock wave accompanies the formation and growth of the cavity, driven by vaporization and expansion at the point of pulse impact. The collapse phase is relatively slow, Figure 3 , and the rate of the energy released in the collapse phase is low. With ultrasonic cavitation, the cavity forms more slowly due to tensile stress on the liquid in the expansion phase, and energy release occurs as it becomes unstable and collapses under the pressure force of the compression phase of the sonic wave. ${ }^{6}$ The shock wave is emitted on collapse, ${ }^{6,22,23}$ and thermal effects in the liquid do not play an important role, due to the large thermal heat capacity of water. ${ }^{20}$ We note that even if a transient temperature rise existed, the crystal nucleation still could take place at some distance of the imploding cavity. A time delay between the original excitation and nuclei formation would therefore also occur with ultrasound.

Further work will be done to better characterize the optical disturbance in Figure 5a and correlate it with the bubble dynamics. Experiments with different laser pulse energies will be useful in this context.

\section{CONCLUSIONS}

Using high-speed photography, the formation of crystal nuclei in supersaturated solutions of $\left(\mathrm{NH}_{4}\right)_{2} \mathrm{SO}_{4}$ and $\mathrm{KMnO}_{4}$ was observed in the wake of a single forming, expanding, and collapsing cavity created by irradiation with a focused laser pulse. The formation and subsequent collapse of the bubble takes about $200 \mu$ s, while the appearance of identifiable small crystals in the vicinity of the cavity occurs on a time scale of seconds following the pulse, although already after $30-50 \mu \mathrm{s}$ small optical disturbances were observed at the same locations where the crystals appeared in the later stage.

The crystals nucleated in these experiments appeared on a ring of diameter $\sim 70 \mu \mathrm{m}$ that was centered at the point of impact of the laser pulse. At its largest extent, the diameter of the disk-shaped cavity was $1400 \mu \mathrm{m}$. At the point where the cavity approaches its maximum diameter, $50 \mu \mathrm{s}$ after the pulse, the optical disturbances appear around the center, and the position of the disturbance corresponds to the ring on which crystals are later seen. The disturbance suggests differences in refractive index and is directly related with the stage at which crystal nucleation initially takes place.

The course of the cavity dynamics observed corresponds closely with the formation and collapse of vapor cavities in microtubes under similar conditions of laser irradiation. Modeling studies ${ }^{9,19}$ for the latter indicate that thermal effects are important in determining the cavity dynamics, with the implication that temperature transients up to $180^{\circ} \mathrm{C}$ occur on impulse at the start of cavity formation. These transients set up the vaporization that leads to the cavity, and heat dissipation determines the dynamics of subsequent collapse. The nature and sequence of these events differ from the dynamics associated with the formation and collapse of ultrasonically induced cavities.

\section{AUTHOR INFORMATION}

\section{Corresponding Author}

*E-mail: a.soare@tudelft.nl.

\section{ACKNOWLEDGMENT}

The authors thank Yoshi Tagawa for many discussions and helpful suggestions. Funding for P.W.C. was provided by NWO via 
a "Bezoekersbeurs” Fellowship. BASF, BP, PSe and Delft Research Centre for Sustainable Industrial Processes are acknowledged for their financial support.

\section{REFERENCES}

(1) Mullin, J. W. Crystallization, 4th ed.; Butterworth-Heinemann: Oxford, 2001.

(2) Kashchiev, D. Nucleation: Basic Theory with Applications; ButterworthHeinemann: Oxford, 2000; Kashchiev, D.; van Rosmalen, G. M. Cryst. Res. Technol. 2003, 38(7-8), 555-574.

(3) Ruecroft, G.; Hipkiss, D.; Ly, T.; Maxted, N.; Cains, P. W. Org. Process Res. Dev. 2005, 9 (6), 923-932.

(4) McCausland, L. M.; Cains, P. W. Biotech. Genet. Eng. Rev. 2004, 21, 3-10; Chem. Ind. 2003, No. 9, 15-18. McCausland, L. M.; Cains, P. W.; Martin, P. D. Chem. Eng. Progr. 2001, 97 (7), 56-61. McCausland, L. M.; Cains, P. W.; Maxwell, M. Chem.-Ing.-Tech. 2001, 73 (6), 717-718.

(5) Young, F. R. Cavitation, 2nd ed.; Imperial College Press: London, 1999.

(6) Brenner, M. P.; Hilgenfeldt, S.; Lohse, D. Rev. Mod. Phys. 2002, 74 (2), 425-484. Flannigan, D. J.; Suslick, K. S. Nature 2005, 434 (7029), 52-55. Didenko, Y. T.; Suslick, K. S. Nature 2002, 418 (6896), 394-397.

(7) Wohlgemuth, K.; Kordylla, A.; Ruether, F.; Schembecker, G. Chem. Eng. Sci. 2009, 64, 4155-4163.

(8) Gaitan, D. F.; Crum, L. A.; Church, C. C.; Roy, R. A. J. Acoust. Soc. Am. 1992, 91 (6), 3166-3183.

(9) Sun, C.; Can, E.; Dijkink, R.; Lohse, D.; Prosperetti, A. J. Fluid. Mech. 2009, 632, 5-16.

(10) Nakamura, K.; Hosokawa, Y.; Masuhara, H. Cryst. Growth Des. 2007, 7 (5), 885-889.

(11) Garetz, B. A.; Aber, J. E.; Goddard, N. L.; Young, R. G.; Myerson, A. S. Phys. Rev. Lett. 1996, 77 (16), 3475-3476.

(12) Yoshikawa, H. Y.; Murai, R.; Maki, S.; Kitatani, T.; Sugiyama, S.; Sazaki, G.; Adachi, H.; Inoue, T.; Matsumura, H.; Takano, K; Murakami, S.; Sasaki, T.; Mori, Y. Appl. Phys. A: Mater. Sci. Process. 2008, 93, 911-915.

(13) Zaccaro, J.; Matic, J.; Myerson, A. S.; Garetz, B. A. Cryst. Growth Des. 2000, 1 (1), 5-8. Garetz, B. A.; Matic, J.; Myerson, A. S. Phys. Rev. Lett. 2002, 89 (17), 175501.

(14) Alexander, A. J.; Camp, P. J. Cryst. Growth Des. 2009, 9 (2), 958-963.

(15) Duffus, C.; Camp, P. J.; Alexander, A. J. J. Am. Chem. Soc. 2009, 131, 11676-11677.

(16) Lindinger, B.; Mettin, R.; Chow, R.; Lauterborn, W. Phys. Rev. Lett. 2007, 99 (4), 045701.

(17) Borkent, B. M.; Arora, M.; Ohl, C. D.; De Jong, N.; Versluis, M.; Lohse, D.; Morch, K. A.; Klaseboer, E.; Khoo, B. C. J. Fluid Mech. 2008, 610, 157-182.

(18) Mullin, J. W.; Chakraborty, M.; Mehta, K. J. Appl. Chem. 1970, $20,367-371$.

(19) Quinto-Su, P. A.; Lim, K. Y.; Ohl, C. D. Phys. Rev. E 2009, 80, 047301.

(20) Kamath, V.; Prosperetti, A.; Egolfopoulos, F. N. J. Acoust. Soc. Am. 1993, 94, 248-260.

(21) Virone, C.; Kramer, H. J. M.; van Rosmalen, G. M.; Stoop, A. H.; Bakker, T. W. J. Cryst. Growth 2006, 294, 9-15.

(22) Roy, R. A. Ultrason. Sonochem. 1994, 1, S5-S6.

(23) Pecha, R; Gompf, B. Phys. Rev. Lett. 2000, 84 (6), 1328-1330. Gompf, B.; Pecha, R. Phys. Rev. E 2000, 61 (5), 5253-5256. 